\title{
Note on semiclassical uncertainty relations
}

\author{
F. Olivares ${ }^{1}$, F. Pennini ${ }^{1,2}$, G.L Ferri ${ }^{3}$, A. Plastino ${ }^{2}$ \\ ${ }^{1}$ Departamento de Física, Universidad Católica del Norte, Av. Angamos 0610, Antofagasta, Chile \\ ${ }^{2}$ Instituto de Física La Plata-CCT-CONICET, Fac. de Ciencias Exactas, \\ Universidad Nacional de La Plata, C.C. 67, 1900 La Plata, Argentina \\ ${ }^{3}$ Facultad de Ciencias Exactas, Universidad Nacional de La Pampa, Peru y Uruguay, Santa Rosa, La Pampa, Argentina
}

(Received on 18 February, 2009)

An important manifestation of the Uncertainty Principle, one of the cornerstones of our present understanding of Nature, is that related to semiclassical localization in phase-space. We wish here to add some notes on the subject with reference to the canonical harmonic oscillator problem, with emphasis in the concepts of semiclassical Husimi distributions, the associated Wehrl entropy, escort distributions, and Fisher's information measure.

Keywords: Husimi distributions, Fisher information, Localizability in phase-space

\section{INTRODUCTION}

It is well known that the uncertainty principle poses a strict bound to semiclassical localizability in phase-space, namely, $\hbar$, instead of the purely quantal $\hbar / 2$ [1-3]. We will here revisit the subject by recourse to concepts like escort distributions of a given order [4] and Fisher's information [5]. To such an end we will utilize the semi-classical methodology detailed, for instance, in Ref. [6], for applying/relating the "escort distribution" concept, joining it with that of information measures expressed in phase-space vocabulary.

Now, the oldest and most elaborate phase-space (PS) formulation of quantum mechanics is that of Wigner [7-9]: to every quantum state a PS function (the Wigner one) can be assigned. This phase-space function can assume negative values so that it is considered a quasi-probability density. This "negative-values' aspect" was circumvented by Husimi [10] (among others), in terms of the so-called Husimi probability distributions $\mu(x, p)$ [11]. (Note that whole of quantum mechanics can be completely reformulated in Husimiterms $[12,13]$.) The distribution $\mu(x, p)$ can be regarded as a "smoothed Wigner distribution" [8]. Indeed, $\mu(x, p)$ is a Wigner-distribution $D_{W}$, smeared over an $\hbar$ sized region (cell) of phase-space [2]. The smearing renders $\mu(x, p)$ a positive function, even if $D_{W}$ does not have such a character. The semi-classical Husimi probability distribution refers to a special type of probability: that for simultaneous but approximate location of position and momentum in phase-space [2]. We will in this communication highlight special aspects of $q$-escort-generalizing Husimi functions so as to show how to improve on this smearing-degree by diminishing the above referred to cell-size $\hbar$. We are particularly motivated by the fact that gaining insight into the emergence of classical behavior (here by recourse to semiclassical ideas) is one of the most attended to present physics' problems [14]. Note also that the subject of phase-space localization is of great relevance in the field of Quantum Chaos (see, for example, [15-18] and references therein).

\section{THE CONCEPT OF ESCORT DISTRIBUTION}

Consider two (normalized) probability distributions $f(x)$, $f_{q}(x)$, and an "operator" $\hat{O}^{q}$ linking them in the fashion

$$
f_{q}(x)=\hat{O}^{q} f(x)=\frac{f(x)^{q}}{\int \mathrm{d} x f(x)^{q}} .
$$

We say that $f_{q}(x)$ is the order $q$-associated escort distribution of $f$, with $q \in \Re$. Often, $f_{q}$ is often able to discern in better fashion than $f$ important details of the phenomenon at hand $[4,6]$.

The expectation value of a quantity $\mathcal{A}$ evaluated with a $q$-escort distribution will be denoted by $\langle\mathcal{A}\rangle_{f_{q}}$. For some physical applications of the concept in statistical mechanics see, for instance (not an exhaustive list), [6, 19-21], and references therein. For physicists, the fundamental reference on escort distributions is [4].

\section{HUSIMI DISTRIBUTIONS AND THEIR WEHRL ENTROPIES}

We review now some material described in detail in Ref. [21], in particular with reference to information instruments expressed in phase-space parlance. A main tool is that called the semi-classical Wehrl entropy $W$, a measure of phasespace localization [1,22] expressed via coherent states $|z\rangle$ $[2,23]$. Coherent states are eigenstates of a general annihilation operator $\hat{a}$, appropriate for the problem at hand [23-25], i.e.,

$$
\hat{a}|z\rangle=z|z\rangle
$$

where $z$ is a complex combination of the phase-space coordinates $x, p$ ( $\hat{a}$ is not Hermitian),

$$
z=z(x, p)=A x+i B p
$$

with $A, B$ being $\hat{a}$-depending constants. The Wehrl entropy definition reads

$$
W=-\int \mathrm{d} \Omega \mu(x, p) \ln \mu(x, p), \quad \mathrm{d} \Omega=\mathrm{d} x \mathrm{~d} p / 2 \pi \hbar
$$

clearly a Shannon-like measure [26] to which MaxEnt considerations apply. $W$ is cast in terms of distribution functions $\mu(x, p)$ commonly referred to as Husimi distributions [10]. As 
a measure of localization in phase-space, $W$ exhibits a lower bound demonstrated by Lieb with reference to the harmonic oscillator coherent states [27]

$$
W \geq 1
$$

on which we will elaborate below.

In turn, Husimi's $\mu$ 's are the diagonal elements of the density operator $\hat{\rho}$, that yields all the available physical information concerning the system at hand [28], in the coherent state basis $\{|z\rangle\}[23]$, i.e.

$$
\mu(x, p) \equiv \mu(z)=\langle z|\hat{\rho}| z\rangle .
$$

These are "semi-classical" phase-space distribution functions associated to the system's $\hat{\rho}$ [23-25]. The distribution $\mu(x, p)$ is normalized in the fashion

$$
\int \mathrm{d} \Omega \mu(x, p)=1
$$

It is shown in Ref. [29] that in the harmonic oscillator case the associated Husimi distribution is

$$
\mu(x, p) \equiv \mu(z)=\left(1-e^{-\beta \hbar \omega}\right) e^{-\left(1-e^{-\beta \hbar \omega}\right)|z|^{2}},
$$

with $\beta=1 / k_{B} T, T$ the temperature, which leads to a pure Gaussian form in the $T=0$-limit. It is also obvious that the $q$-escort Husimi distribution $\gamma_{q}(x, p)$ will be

$$
\gamma_{q}(x, p)=\hat{O}^{q} \mu(x, p)=\frac{\mu(x, p)^{q}}{\int \mathrm{d} \Omega \mu(x, p)^{q}},
$$

which for the $\mathrm{HO}$ reduces to

$$
\gamma_{q}(x, p)=q\left(1-e^{-\beta \hbar \omega}\right) \exp \left(-q\left(1-e^{-\beta \hbar \omega}\right)|z|^{2}\right) .
$$

Its associated Wehrl's measure becomes

$$
W_{q}=-\int \mathrm{d} \Omega \gamma_{q}(x, p) \ln \gamma_{q}(x, p) .
$$

i.e.,

$$
W_{q}=W-\ln q,
$$

as it was shown in Ref. [3], and the analytic expression for $W$ is [2]

$$
W=1-\ln \left(1-e^{-\beta \hbar \omega}\right)
$$

By requiring $W_{q}$ to range between 0 and 1 we easily ascertain that, at $T=0$,

$$
1 \leq q \leq e
$$

The $W_{q}$-requirement arises from wanting it to have a lower upper bound than Liebs's.

\section{PARTICIPATION RATIO}

We start here presenting our results. The question of interest now is to further refine the range of possible values that $q$ may adopt. We introduce to this effect the "semi-classical" version of a well-known quantum concept, that of participation ratio [30], here associated to the $q$-escort Husimi distribution $\gamma_{q}$

$$
\mathcal{R}_{q}=\frac{1}{\int \frac{\mathrm{d}^{2} z}{\pi} \gamma_{q}(z)^{2}}
$$

which, if we explicitly compute, reads

$$
\mathcal{R}_{q}=\frac{2}{q\left(1-e^{-\beta \hbar \omega}\right)} .
$$

We can note that $\mathrm{d}^{2} z / \pi \equiv \mathrm{d} x \mathrm{~d} p / 2 \pi \hbar$. When the temperature $T=0$ we have $\mathcal{R}_{q}=2 / q$ and, when $T$ goes to infinity, obviously $\mathcal{R}_{q}$ goes to infinity too. Since it is well-known that [30] $\mathcal{R}_{q} \geq 1$, this immediately entails

$$
q \leq 2
$$

In view of (14) we have now a better-defined range of values for $q$, namely, the new range

$$
1 \leq q \leq 2
$$

\section{SEMICLASSICAL FISHER INFORMATION IN PHASE-SPACE}

The last years have witnessed a great deal of activity revolving around physical applications of Fisher's information measure (FIM) [5]. FIM provides one with a powerful variational principle, the extreme physical information one, that yields most of the canonical Lagrangians of theoretical physics [5], characterizing also in quite a proper fashion an "arrow of time", alternative to the one associated with Boltzmann's entropy [31, 32]. The classical Fisher information associated with translations of a one-dimensional observable $x$ with corresponding probability density $\rho(x)$ is [33]

$$
I_{x}=\int \mathrm{d} x \rho(x)\left[\frac{\partial \ln \rho(x)}{\partial x}\right]^{2},
$$

and the Cramer-Rao inequality is given by [33]

$$
\Delta x \geq I_{x}^{-1}
$$

where $\Delta x$ is the variance for the stochastic variable $x$ which is of the form [33]

$$
\Delta x^{2}=\left\langle x^{2}\right\rangle-\langle x\rangle^{2}=\int \mathrm{d} x \rho(x) x^{2}-\left(\int \mathrm{d} x \rho(x) x\right)^{2} .
$$

An original, compact expression is here advanced for the "semiclassical" Fisher information measure, which can be easily derived from the Wehrl-methodology described in [29]. The new result reads

$$
I_{F}^{s c}=\frac{1}{4} \int \frac{\mathrm{d}^{2} z}{\pi} \mu(z)\left\{\frac{\partial \ln \mu(z)}{\partial|z|}\right\}^{2},
$$


so that inserting the $\mu$-expression into (22) we find the analytic form

$$
I_{F}^{s c}=1-e^{-\beta \hbar \omega},
$$

leading to the following limits:

$$
\begin{aligned}
& \text { For } T \rightarrow 0 \text { one has } I_{F}^{s c}=1 \\
& \text { For } T \rightarrow \infty \text { one has } I_{F}^{s c}=0,
\end{aligned}
$$

as it should be expected. As for the associated escort-Fisher measure $I_{q}$ one easily gets

$$
I_{q}=\frac{1}{4} \int \frac{\mathrm{d}^{2} z}{\pi} \gamma_{q}(z)\left\{\frac{\partial \ln \gamma_{q}(z)}{\partial|z|}\right\}^{2},
$$

which using (10) leads to

$$
I_{q}=q\left(1-e^{\beta \hbar \omega}\right)=q I_{F}^{s c},
$$

entailing that $0<I_{q} \leq q$.

\subsection{Fisher uncertainties}

It was shown in Ref. [34] that variances for $x$ and $p$ evaluated with de Husimi distribution yield a Fisher-weighted uncertainty relation

$$
I_{F}^{S c} \Delta_{\mu} x \Delta_{\mu} p=\hbar .
$$

We wish here to show that this relations is invariant under the escort transformation. In other words, this is tantamount to replacing $\mu$ for $\gamma_{q}$ in the evaluation of the variances and also $I^{s c}$ for $I_{q}$. Now, from Ref. [3] we know that

$$
\Delta_{\gamma_{q}} x \Delta_{\gamma_{q}} p=\frac{\hbar}{q\left(1-e^{-\beta \hbar \omega}\right)}
$$

so that, using also Eq. (25) we easily obtain the (original in this context) relation

$$
I_{q} \Delta_{\gamma_{q}} x \Delta_{\gamma_{q}} p=\hbar,
$$

which is the promised invariance. Contrariwise, removing the Fisher measure from the above relations it destroys this invariance and yields interesting results that we discuss below.

\subsection{Husimi uncertainties}

Husimi-mean values $\left\langle x^{2}\right\rangle,\left\langle p^{2}\right\rangle,\langle x\rangle$, and $\langle p\rangle$ have been computed in [3], reading

$$
\begin{aligned}
\left\langle x^{2}\right\rangle_{\mu} & =\frac{2 \sigma_{x}^{2}}{1-e^{-\beta \hbar \omega}}, \\
\left\langle p^{2}\right\rangle_{\mu} & =\frac{2 \sigma_{p}^{2}}{1-e^{-\beta \hbar \omega}}
\end{aligned}
$$

and

$$
\langle x\rangle_{\mu}=\langle p\rangle_{\mu}=0,
$$

with $\sigma_{x}=(\hbar / 2 m \omega)^{1 / 2}$ and $\sigma_{p}=(\hbar m \omega / 2)^{1 / 2} . T$-dependent uncertainty relations for the harmonic oscillator straightforwardly follow now in the fashion

$$
\Delta_{\mu} x \Delta_{\mu} p=\frac{\hbar}{1-e^{-\beta \hbar \omega}},
$$

that, at zero-temperature, lead to the well-known result referred to in the Introduction (and that we wish to improve upon), i.e.,

$$
\left(\Delta_{\mu} x \Delta_{\mu} p\right)_{T=0}=\hbar
$$

We can evaluate these variances again using the $\gamma_{q}$-escort distribution, being easily led to

$$
\begin{aligned}
\left\langle x^{2}\right\rangle_{\gamma_{q}} & =\frac{\left\langle x^{2}\right\rangle}{q}=\frac{2 \sigma_{x}^{2}}{q\left(1-e^{\beta \hbar \omega}\right)}, \\
\left\langle p^{2}\right\rangle_{\gamma_{q}} & =\frac{\left\langle p^{2}\right\rangle}{q}=\frac{2 \sigma_{p}^{2}}{q\left(1-e^{\beta \hbar \omega}\right)}, \\
\langle x\rangle_{\gamma_{q}} & =\langle p\rangle_{\gamma_{q}}=0,
\end{aligned}
$$

which finally yield the new uncertainty relationship

$$
\Delta_{\gamma_{q}} x \Delta_{\gamma_{q}} p=\frac{\Delta_{\mu} x \Delta_{\mu} p}{q}=\frac{\hbar}{q\left(1-e^{\beta \hbar \omega}\right)} .
$$

When the temperature goes to zero we find that

$$
\left(\Delta_{\gamma_{q}} x \Delta_{\gamma_{q}} p\right)_{T=0}=\frac{\hbar}{q}
$$

which sensibly improves the typical, cell of size $\hbar$ semiclassical power, and is also better than the $\hbar / \sqrt{q}$ result of [3]. Indeed, we recover the pure quantum result for the maximum permissible $q$-value of 2 .

\section{CONCLUSIONS}

We summarize now our main results. We have advanced three original expressions, i.e., Eqs. (14), (22), (25) and (29). We have observed the invariance of the Fisher uncertainties under the escort transformation and, finally, we have shown that with the help of the escort distributions one is able to reobtain the canonical Heisenberg's uncertainties at the semiclassical level, thus clearly exhibiting the power of the escort-concept.

Acknowledgment F. Pennini and F. Olivares are grateful for the partial financial support by FONDECYT 1080487. 
[1] A. Wehrl, Rep. Math. Phys. 16, 353 (1979).

[2] A. Anderson and J.J. Halliwell, Phys. Rev. D 48, 2753 (1993).

[3] F. Pennini, A. Plastino, G. L. Ferri, F. Olivares, Phys.Lett. A 372, 4870 (2008).

[4] C. Beck and F. Schlögl, Thermodynamics of chaotic systems, Cambridge University Press, Cambridge, England, (1993).

[5] B.R. Frieden, Physics from Fisher information, (Cambridge University Press, Cambridge, England, 1998); Science from Fisher information, (Cambridge University Press, Cambridge, England, 2004).

[6] F. Pennini, A. Plastino, G.L Ferri, Physica A 383, 782 (2007).

[7] E.P. Wigner, Phys. Rev. 40, 749 (1932).

[8] H.W. Lee, Phys. Rep. 259, 147 (1995).

[9] J.J. Wlodarz, Int. J. Theor. Phys. 42, 1075 (2003).

[10] K. Husimi, Proc. Phys. Math. Soc. Japan 22, 264 (1940).

[11] Note that the Husimi distribution function is not, strictly speakig, a probability density because the marginal distribution on each variable is not the squared modulus of a wave function.

[12] R.F. O’ Connel and E.P. Wigner, Phys. Lett. A 85, 121 (1981).

[13] S.S. Mizrahi, Physica A 127, 241 (1984).

[14] W.H. Zureck, Rev. Mod. Phys. 75, 715 (2003).

[15] H.J. Korsch and W. Leyes, New Journal of Physics 4, 62 (2002).

[16] M. Baranger, M.A.M. de Aguiar, F. Keck, H.J. Korsch, and B. Schellhaass, J. Phys. A: Math. Gen. 34, 7227 (2001).

[17] Ph. Jacquod and R.S. Whitney, arXiv:cond-mat/0512662.

[18] J.L. Schoendorff, H.J. Korsch, and N. Moiseyev Europhys. Lett. 44, 290 (1998).
[19] F. Pennini, A.R. Plastino, and A. Plastino, Physica A 258, 446 (1998).

[20] R.P. Di Sisto, S. Martinez, R.B. Orellana, A.R. Plastino, A. Plastino, Physica A 265, 590 (1999).

[21] F. Pennini and A. Plastino, Phys. Lett. A 326, 20 (2004)

[22] S. Gnuzmann, K. Życzkowski, J. Phys. A 34, 10123 (2001).

[23] R.J. Glauber, Phys. Rev. 131, 2766 (1963).

[24] J.R. Klauder and B.S. Skagerstam, Coherent states, World Scientific, Singapore, (1985).

[25] J. Schnack, Europhys. Lett. 45, 647 (1999).

[26] E. T. Jaynes in Statistical Physics, ed. W. K. Ford (Benjamin, New York, 1963); A. Katz, Statistical Mechanics, Freeman, San Francisco, (1967).

[27] E.H. Lieb, Commun. Math. Phys. 62, 35 (1978).

[28] R.K. Pathria, Statistical Mechanics Pergamon Press, Exeter, (1993).

[29] F. Pennini and A. Plastino, Phys. Rev. E 69, 057101 (2004).

[30] J. Batle, A.R. Plastino, M. Casas, and A. Plastino, J. Phys. A: Math. Gen. 35, 10311 (2002); W.J. Munro, D.F.V. James, A.G. White, and P.G. Kwiat, Phys. Rev. A 64, 0303202 (2003).

[31] A.R. Plastino and A. Plastino, Phys. Rev. E 54, 4423 (1996).

[32] A. Plastino, A.R. Plastino and H.G. Miller, Phys. Lett. A 235 , 129 (1997).

[33] Michael J.W Hall, Phys. Rev. A 62, 012107 (2000).

[34] F. Pennini, A. Plastino, G.L. Ferri, Phys. Lett. A, 359, 14 (2006). 